\title{
Neurosciences in criminology
}

\author{
Franco Posa ${ }^{1}$ and Gabriele A. Losa ${ }^{2}$ \\ ${ }^{1}$ Clinical studies in criminology, Sementina, Switzerland \\ ${ }^{2}$ Institute for Interdisciplinary Scientific Studies. Locarno, Switzerland
}

\begin{abstract}
Summary
Neuroscience applications to criminology have acquired increasing scientific and legal value thanks to recent studies of the anatomy and metabolic activity of the brain. The customary clinical methods of investigation of cerebral metabolism are now applied to scientific studies of brain activity, with the aim of objectively identifying the active areas in subjects with antisocial or criminal behavior. Modern criminal neurosciences seek to identify the relationship between the brain and the mind, searching for evidence of how the mind can emerge from its biological substrate. Tools such as fMRI or SPECT are currently used for: metabolic investigation of different brain areas involved in the genesis of some behavioral traits, and identification of the areas involved in the construction of lies, or in any particular psychiatric condition.
\end{abstract}

\section{Part I: General introduction to forensic neurosciences}

The body of knowledge developed in the last decades thanks to neuroscience research reflects an interest beyond biomedical research and clinical applications: it reaches further, in the direction of the juridical sector. The evolution of the new neurosciences, and their applications' expansion, has culminated in the scientific interaction between neuroscience and law, with the inclusion of all figures involved in this process: first of all criminals, but also victims and witnesses.

Could a faulty neuronal circuit justify the abnormal behavior of a subject, while eluding his will?

Can we speak of free will when we know from molecular genetics that an allele, versus another, significantly increases the probability of seeking extreme situations even outside legality?

The evolution of neurosciences in the last twenty years has led to a drastic change in the concept of human mind and behavior. Considering the outcomes of neuroscience research, the debate remains whether an individual is truly free in his/her choices and actions, or rather predetermined by anatomical elements. The millenary question of free will becomes once again open for discussion. Numerous queries arise at the crossing where sciences meet philosophy and law.

This integration results in a new science with its associated emerging research, the so-called "juridical neurosciences". The term 'neuroscience' is currently used in criminology to refer to a group of diverse scientific disciplines that share a common goal despite being heterogeneous: to understand brain mechanisms that result in human behaviors and phenomena, including the most complex ones, considered inaccessible to scientific inquiry until recently. Modern criminal neuroscience seeks to identify the relationship between brain and mind, in order to obtain a scientifically recognizable indication of how the mind emerges from its biological substrate.

The existing definitions of neuroscience are evidence of the rapid evolution of some important principles. We used to believe human behavior is exclusively the result of complex social interactions, its understanding is therefore uniquely possible in light of environmental and social influences. The "standard model" stated that only society and environment could explain a socially inappropriate behavior. In this regard, it is worth remembering that Durkheim, a sociology scholar, believed the determining cause of a social event should be sought among antecedent social facts, not among the layers of individual consciousness. Until a few years ago, studies of the brain were limited to pathology; today we are facing a neuroscience evolution in the understanding of how human beings are able to think, decide and act, not only in pathological conditions.

In recent years behavioral genetics, sociology, evolutionary psychology, neurochemistry, and cognitive neuroscience have individually presented and highlighted correlations between organism and behavior. Hence the definition of legal neuroscience, or what has been described so far as an ambitious attempt to group different areas of research with the aim of applying neuroscientific methods to the study and practice of law. This definition inherently contains a first difficulty of adapting legal neuroscience to each national system after it is developed in an international perspective. Another widely used definition is forensic neuroscience, a study of the link between relevant neuroscientific data for the purpose of a judicial assessment. The most difficult objective is ensuring the validity of its scientific tools, and the suitability of its theories and methods in providing valid scientific evidence in a trial setting.

In recent years scientific contributions have acquired increasing value along with the belief that it is now possible, through the study of neuroanatomy, to measure the structure of the brain and its functions, and to identify structural abnormalities in temporal and limbic areas, the hippocampus, the amygdala and the frontal lobe.

Correspondence to: Gabriele A. Losa, Institute for Interdisciplinary Scientific Studies. Locarno, Switzerland, E-mail: gabriele.losa@bluewin.ch

Key words: imputability, functional magnetic resonance, neuroscience, SPECT, neuroimaging, brain, metabolism, BOLD, criminology

Received: July 29, 2016; Accepted: August 27, 2016; Published: August 30, 2016 
The principal means of investigation that have allowed brain exploration for clinical purposes (neuroimaging) for some years now are: computer analysis of the EEG, with a selective mapping of the electrical activity of specific and well-defined brain areas; computerized axial tomography (CAT); functional magnetic resonance imaging (fMRI); positron emission tomography (PET), magnetoencephalography (MEG); single photon emission computer tomography (SPECT); as well as acquisitions of neurotransmission and neuromodulation activity; and last but not least, the study of molecular neurobiology. SPECT in particular appears to be the proper tool for identifying cerebral perfusion with the help of radiopharmaceuticals, due to the fact they are able to cross the blood-brain barrier. This method highlights and quantifies any change in Regional-Cerebral Blood Flow $(\mathrm{rCBF})$ in the brain region involved by a specific external stimulation. In view of the fact that neurons do not have a readily available source of energy, increasing blood flow is fundamental when greater amounts of oxygen and metabolites are necessary in a specific brain region. The correlation between blood flow and cerebral metabolism has laid the scientific foundation for functional studies of the brain. In SPECT, reconstructed images generally have a resolution of $64 \times 64$ pixels, or $128 \times 128$, with a pixel size varying between 3 and $6 \mathrm{~mm}$. The number of acquired projections is set to be approximately equal to the width of the resulting image. Generally, images reconstructed after processing will be of lower resolution, will have more noise than planar images, and will be more susceptible to artifacts. The scan takes a variable amount of time, and it is essential that during the process of acquisition the patient do not move. This is one of the most significant limitations, especially when doing brain studies for forensic purposes, where the subject is exposed to external verbal or visual stimuli (experimental technique). Movement can cause a significant reduction in the quality of reconstructed images, even though image reconstruction techniques compensate for movement by reducing artifacts. The iterative reconstruction technique obtained with an alternative algorithm is attracting increasing interest, because it is less sensitive and thus better protected against unwanted artifacts. The attenuation of gamma rays in the human body produces a significant underestimation of deep tissue when compared with more superficial tissues. An optimal correction is possible by measuring attenuation values. Modern SPECT are provided with an X-ray scanner used for CT; the images can then be reworked with compatible CT systems, and more anatomical details become available. The gamma-emitting tracer used in functional neuroimaging is $99 \mathrm{mTc}-\mathrm{HMPAO}$ (hexamethylpropylene amine oxime). The $99 \mathrm{~m}$ is a metastable nuclear isomer able to emit gamma rays, which are detected by a gamma camera. When associated to the HMPAO, 99mTc is absorbed by brain tissue in proportion to blood flow; in this manner cerebral blood flow can be detected by the gamma camera. Blood flow to the brain is directly related to the metabolism of the activated brain region, and to the energy used by the brain mass. The $99 \mathrm{mTc}-\mathrm{HMPAO}$ tracer is administered in order to identify each region's brain activity, and is already a widely used technique in the study of dementias. The neuroforensic purpose of this technique is to visualize the subject's baseline brain activity, and when possible, the relationship between external stimuli (demand, image) and cerebral activity. This type of functional assessment still appears to be experimental, and does not yet have easy interpretation and application. Glucose metabolism in the activated brain also appears to be detectable and quantifiable by PET imaging (positron emission tomography), through quantification of fluorodeoxyglucose (FDG) (Figure 1).

When considering metabolic activity and regional cerebral blood flow, a significant factor is also linked to synaptic activity, which is

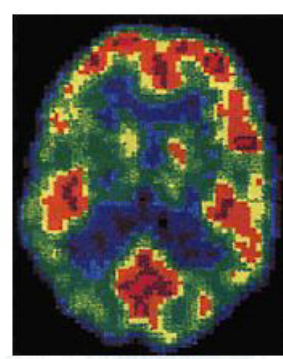

NORMAL

CONTROL

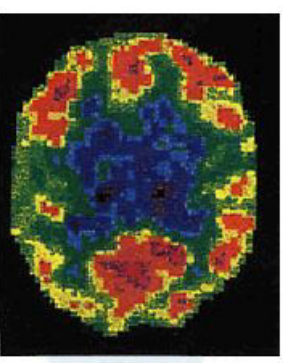

DEPRIVED MURDERER

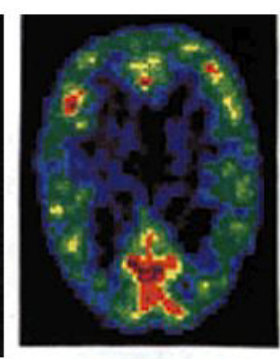

NON-DEPRIVED
Figure 1. These PET scan images illustrate the reduced prefrontal glucose metabolism (upper part of scan) in a murderer lacking psychosocial deprivation (right) compared to a murderer with psychosocial deficits (middle), and one normal prefrontal activation.

mainly mediated by an excitatory neurotransmitter, glutamate. With reference to what has been described so far, it is assumed that astrocytes (glial cells) are one of the mediators involved in the micro-vasodilation mechanism. It is only right to point out that, to date, the use of FDGPET produces images with a spatial resolution of higher anatomical precision.

SPECT studies have produced evidence, with favorable sensitivity and specificity characteristics, of reduced frontal perfusion in patients with antisocial behavior (Soderstrom et al., 2002). SPECT is particularly sensitive and specific in studies carried out during a critical stage (crisis or post critical phase) of epilepsy. This feature is not to be underestimated, and of particular importance in the context of neuroimaging related to all behavior, not exclusively antisocial (post critical stage in aggressive stimulation). The described methodology is significantly influenced by the use of exogenous substances (tobacco, alcohol) by the subject under examination. It is also crucial to note the interference of external stimulating visual and auditory factors: their description is beneficial to identify the relationship between auditory, visual, or verbal stimuli, and the activity of a respective area of the brain. This is a critical feature in imaging used to uncover a relationship between brain area and altered behavior secondary to an administered stimulus. The report generated by SPECT images is prepared by direct observation and qualitative analysis. It is also possible to obtain a semiquantitative analysis by regions of interest (RO) or with statistical parametric systems. The uptake is normalized before making statistical comparisons, by measuring an area not affected by the disruption of metabolic processes, weighing up the data from different subjects with a different basal metabolism, and following with another study after stimulation. The most widely examined regions in the brain are the pons and the cerebellum.

\section{The deoxyglucose method}

The [18F]fluorodeoxyglucose $\left({ }^{18} \mathrm{FDG}\right)$ method for quantitative measurement of rCMRglc was developed approximately 20 years ago, and was initially used with Single Photon Emission

Computerized Tomography (SPECT), and shortly after with PET. This methodologic model, formulated by Louis Sokoloff, derives from a kinetic analysis of the biochemical behavior of 2-deoxy-D-glucose; subsequent modifications of this model allow us today to calculate rCMRglc in vivo in humans. The 2-deoxy-D-glucose (DG) molecule is a glucose analogue, it differentiates itself only for the absence of a carbon atom in the position $\mathrm{C} 2$ of a hydroxyl group -OH; it shares with glucose the same affinity for the transmembrane carrier, by which it is transported bi-directionally through the BBB for hexokinase and glu- 
6-phosphatase, thus becoming a substrate.

Glucose and DG have identical values concerning the affinity between glucose and its transporter (or enzyme), hence for the velocity of transport (or reaction) of glucose from plasma to cerebral tissue and viceversa. The speed of a biochemical reaction is equal to the ratio between product concentration/substrate concentration, hence the ratio between DG-6-P/DG concentrations is equal to the ratio of glucose-6-P/glucose concentrations, and is equal to the net velocity of the hexokinase catalyzed reaction. Unlike glucose-6-P, DG-6-P is not a substrate for the subsequent enzyme in the glycolitic chain (hexophosphate isomerase), therefore cannot be further degraded by a glycolitic process. That is how virtually all DG-6-P produced at the neuronal level by the hexokinase enzyme accumulates in the citoplasm for at least the first 45 minutes, as a direct function of the net catalitic reaction velocity, and indirectly, as a function of neuronal activity given the absence of intra- or extra cellular DG-6-P transporters, the low neuronal activity of the glucose-6-P phosphatase enzyme, and the negligible amount of DG-6-P turning into glycogen. rCMRglc is expressed in glucose micromoles per 100 grams of cerebral tissue per minute $(\mu \mathrm{moli} / 100 \mathrm{~g} / \mathrm{min})$ or in milligrams of glucose per 100 grams of cerebral tissue per minute $(\mathrm{mg} / 100 \mathrm{~g} / \mathrm{min})$. The cerebral values of rCMRglc, $\mathrm{rCBF}$ and $\mathrm{rCMO} 2$ in young adults, are reported in the Table 1 below.

\begin{tabular}{|l|c|c|c|}
\hline & Global cerebral & Grey Matter & White Matter \\
\hline rCMRglc $(\mu \mathrm{moles} / 100 \mathrm{~g} / \mathrm{min})$ & $28-32$ & $28-41$ & $16-19$ \\
\hline $\mathrm{rCBF}(\mathrm{ml} / 100 \mathrm{~g} / \mathrm{min})$ & $41-71$ & $28-58$ & $20-24$ \\
\hline $\mathrm{rCMRO} 2(\mathrm{ml} / 100 \mathrm{~g} / \mathrm{min})$ & $3.5-4$ & $3.1-4.7$ & $1.2-1.9$ \\
\hline
\end{tabular}

Table 1. The cerebral values of rCMRglc, $\mathrm{rCBF}$ and $\mathrm{rCMO} 2$ in young adults

\section{fRMN and cerebral metabolism}

Once again we mention functional Magnetic Resonance Imaging fMRI [1], found to be useful in the presence of abnormalities in frontal lobe activation, during tasks of response inhibition, and processing of emotional stimuli (Müller et al., 2003). Temporal lobes have also been successfully studied during exposure to, and elaboration of words and images carrying an elevated emotional charge (Figure 2).

FMRI deserves particular attention, as it is often used as neuronal functional magnetic resonance imaging, a newly developed neuroimaging technique devoted to identifying cerebral metabolic areas to be examined during a baseline or stimulation study. This method identifies and quantifies the hemodynamic response (change in the oxygen content of the parenchyma and capillaries) in relation to the activity of neurons. At a distance of approximately 1-5 seconds after neural activation, there is an increase in blood flow. At the end of the stimulus, the oxygen peak decreases to levels even lower than pre-stimulus activity. Hemoglobin has a diamagnetic behavior when oxygenated but becomes paramagnetic when oxygen transport is reduced, with a resulting variation in the fMRI signal. The contrast medium used for this study is defined as Blood Oxygenation Level Dependent (BOLD). The increase in BOLD signal derives from a decrease in the oxygenated hemoglobin concentration. Still no clear interpretation is given to the direct relationship between blood supply and neural input-output messages relatable to brain activity. From an electrical point of view the local field potentials provide an indication of integrated electrical activity in relation to blood flow. Action potentials are most directly related to neural communications. The complex nature of the cerebral metabolic mechanisms has not yet made it possible to identify with certainty the relationship between

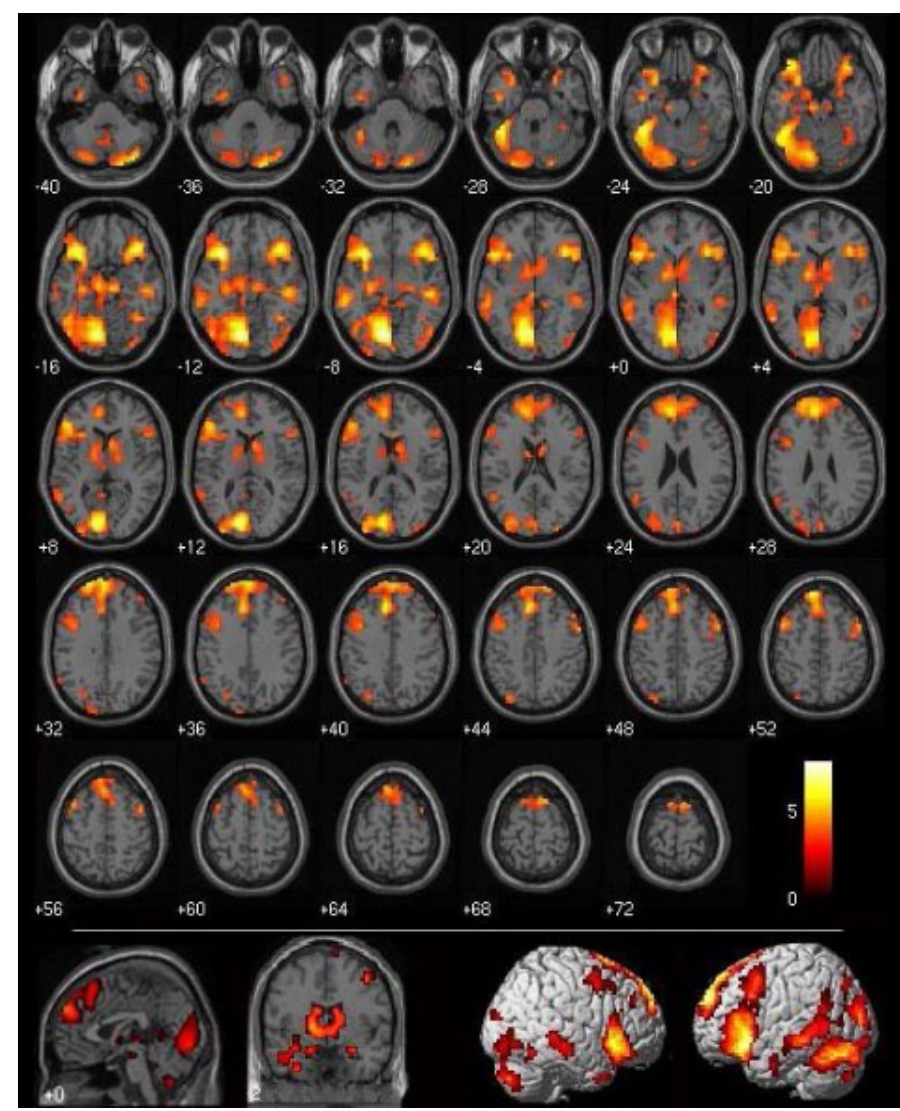

Figure 2. fMRI Images: "How your brain makes moral judgments." Cnn.com. 27 March 2014.

electrical and metabolic activity, a scientific challenge which is not negligible in relation to neuro-criminological interests. To date, the negative BOLD signal has produced an imaging mechanism for ocular dominance columns in the primary visual cortex, with a resolution often inferior to $0.5 \mathrm{~mm}$. The BOLD signal is quantified by measuring a rapid volumetric acquisition with contrast weighted images. Images are captured in a period between one and four seconds, and each Voxel obtained represents a cube of tissue about 3-4 mm per side. The response to stimuli 1-2 seconds apart is discriminated through the event-related method. The described methods, and their integration with neuroanatomical and neurophysiological knowledge, allow us to take into consideraton the explanation of scientific phenomena in relation to human cognition. Criticisms to the above methods are directed to the BOLD signal as an indirect measure of neural activity, and therefore susceptible to the influence of non-neural physiological phenomena. Furthermore, different areas of the brain could have different hemodynamic capacities which are not accurately represented by the general linear model used as a filter for time signals. Finally, functional MRI has unveiled only in recent years its application, of undeniable scientific interest, as "Lie Detector". This application is still under much discussion and wide scientific debate. Basically using the same principles of the classic lie detector, it identifies activation of different brain areas instead of changes in physiological parameters (Figure 3) (Lavazza and Sartori, 2011).

From an experimental point of view, one can see whether a criminal brain reports the truth about an incident, or about details that have characterized a criminal event. The use of functional MRI for these purposes results to be widely opposed by the scientific community, 


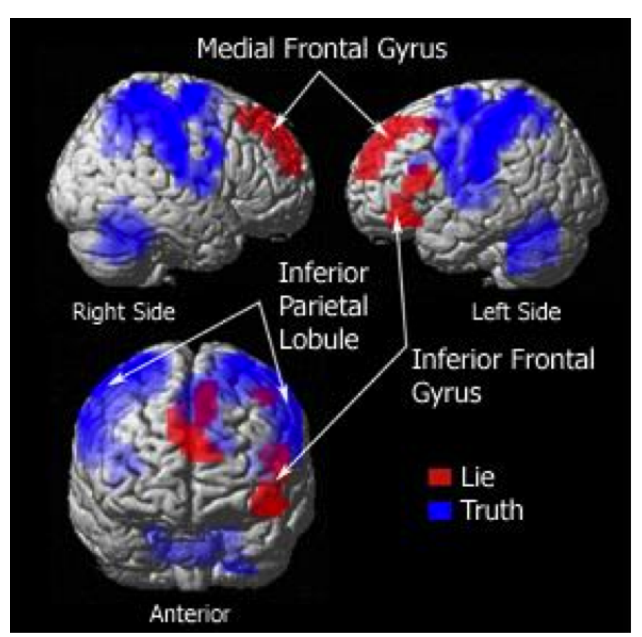

Figure 3. fMRI "Lies and Truth" Map.

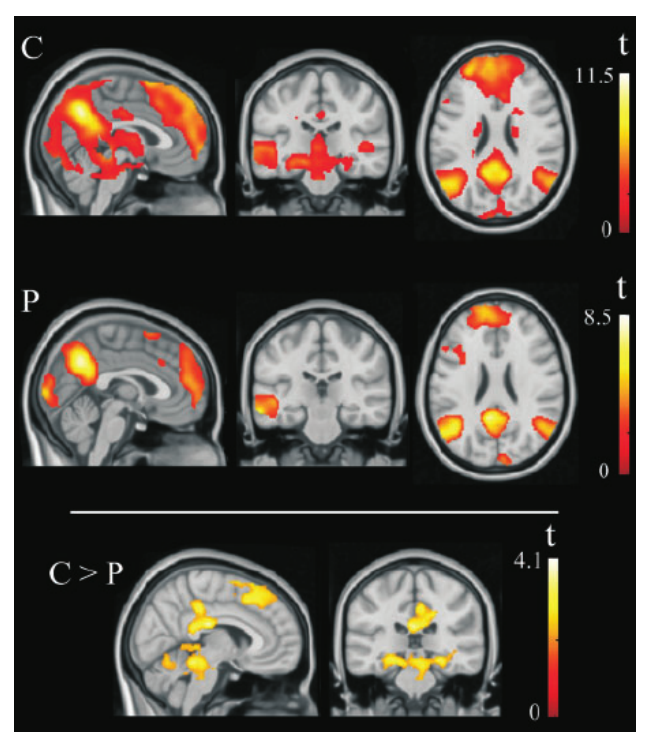

Figure 4. fMRI patterns of brain activation during the moral dilemma task. Compared with control subjects $(\mathrm{C})$, psychopathic individuals $(\mathrm{P})$ showed significantly reduced activation in the medial frontal cortex and the posterior cingulate cortex and additionally in both hippocampus and posterior - inferior midbrain. Right side of the figure corresponds to the right hemisphere for both axial and coronal views.

where this forensic application is seen as disrespectful of the Daubert rule (Simpson, 2008; Marikangas, 2008; Schauer, 2009). In addition, criteria of reliability of scientific results turn out to be different from legal criteria, and these differences require an adaptation which is not easy to apply (Schauer, 2009-2010). Common opinion is that, in order to validate the survey regarding the Lie Detector, it is necessary to reach objectively verifiable targets in terms of biological measures (membranes, receptors) of macroscopically detectable changes on the MRI (Figure 4).

\section{Voxel-based morphometry}

One technique currently used to determine anatomical alterations of the brain is the "Voxel-Based Morphometry" (VBM). This technique allows a comparison (voxel by voxel) of cerebral microstructures, with the possibility to detect and quantify the relationship between gray and white matter in a two-group comparison, starting from the elaboration of images in nuclear magnetic resonance. This technique has been used with success in the exploration of psychopathological conditions defined as functional by classical psychiatry. The evaluation uses a statistical approach known as "statistical parametric mapping (Figure 5)."

In conventional methods brain volume, or the volume of specific areas, is measured by highlighting regions of interest from the developed brain scan, and then calculating the residual volumes. In conventional morphometric determinations it is frequently impossible to map brain areas that are deeply branched, or those with atypical distribution. Volumetric masses with branching characteristics are related through VBM to a computerized anatomical atlas of the brain. Through a process of "smoothing", each voxel allows the representation of 1 voxel and the surrounding 26 voxels (geometric dimension of a cube of $3 \times 3 \times 3$ voxels); at this point it will be imperative to compare the obtained image with other referenced anatomical regions [2]. This is also an opportunity to apply this method to the identification of differences in brain morphology between different subjects, as well as to the observation of comparisons and differences between the two hemispheres of a subject's brain mass, and finally to study asymmetries in the human brain [3].

Some evidence of particular scientific interest has been confirmed by the demonstration of this method's significant sensitivity in highlighting microstructural changes in the gray-white matter relationship in association with personality disorders, thus confirming a significant scientific relationship between psychiatric disorders and brain morphology.

\section{Evoked potentials}

The use of evoked potentials (ERPs), as a technique for exploring brain function without considering its structural anatomical elements, has become a validated technique for investigating cerebral electrical activity correlated to external stimuli or to the execution of administered

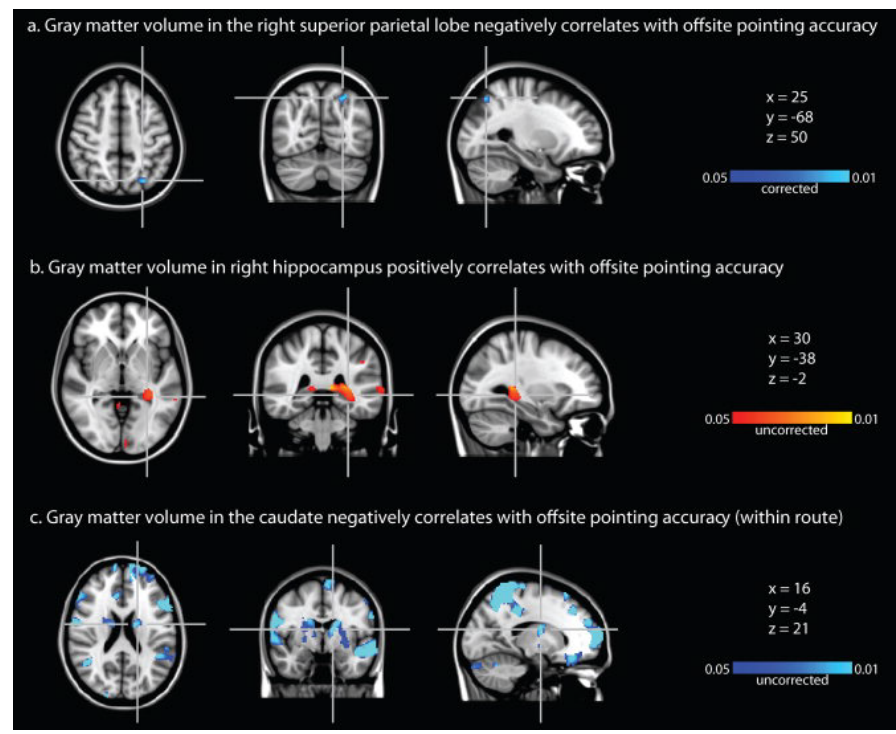

Figure 5. Voxel-Based Morphometry (VBM) results. (a) Negative correlation between gray matter volume in the right superior parietal lobe and offsite pointing accuracy. (b) Positive correlation between gray matter volume in the right hippocampus and offsite pointing accuracy. Note that the increase in gray matter is located in the tail of the right hippocampus. (c) Negative correlation between gray matter volume in the caudate (bilateral) and offsite pointing accuracy. Threshold in (a) is $\mathrm{P}<0.05$ corrected for multiple comparisons across the entire brain; thresholds in (b) and (c) are $\mathrm{P}<0.05$ uncorrected (Psychiatry Neuroimaging Laboratory, Department of Psychiatry, Brigham and Women's Hospital, Harvard Medical School, Boston, MA, USA). 
or given tasks. In view of the new opportunities of investigation described above, one must consider obsolete the subdivision of mental disorders that led to regard mere psychoses (with the exception of psychopathy), neuroses, or personality disorders, as objectively and clinically ascertainable illnesses, due to the lack of objective diagnostic possibilities. Atypical disorders (defined as functional) have been partially described in relation with their neuronal counterparts, and therefore with their genetic basis. To date there is an opportunity to correlate almost any mental disorder with a specific brain alteration of structural or functional origin. Scientific literature provides repeated consensus with regards to the new neurosciences.

The new brain research techniques are bringing forth gradual improvements in terms of reports becoming objective and scientific, with convincing science-based judgments. Neuroimaging techniques study brain activity during emotional stimulation or during different behavioral responses in physiological conditions. Particular attention is paid to the study of activity in the amygdala as a sentinel or emotional computer within the brains complex activity. Equally interesting are the opportunities for a legal response to the exploration of activity in the frontal and prefrontal lobes (orbital and medial areas of the ventral anterior cortex): there is indeed an opportunity to evaluate the neural correlates of consciousness that are involved in the planning of an act, or in controlling one's impulsiveness. The hypotheses regarding the relationship between reduced prefrontal function and predisposition to violence can have different scientific interpretations today thanks to neuroscience. The first hypothesis refers to a reduced prefrontal function at the emotional level leading to the reduction in control over more primitive sections of the brain, such as the limbic system, which is capable of generating primary emotions like anger and rage. The evolved prefrontal cortex is capable of limiting limbic emotions. Reduction or cancellation of these control mechanisms generates the uncontrolled response.

In the second hypothesis, we know from a neurological point of view that prefrontal damage leads to increased susceptibility to accept a risk, generating irresponsibility and failure to respect the rules. The third hypothesis states that frontal damage can lead to personality change, a situation that is widely known in the clinical field, with the proven relationship between invasion of the frontal area by neoformations or trauma, and a corresponding behavioral modification.

Another hypothesis supports the belief that prefrontal damage can cause immaturity, and consequently, poor social standing. The consequence of such condition will be socially unacceptable behavior, with a compromised ability to elaborate non-aggressive solutions in socially difficult situations. In our cognitive hypothesis, limitations in function of the prefrontal cortex can cause the so-called lack of problem solving. This intellectually limiting situation becomes the ground for scholastic, social, professional, and economic failure, all factors evidently predisposing to criminal behavior [6,7]. In the event that neuroscientific evidence identifies normal functioning of the prefrontal cortex in the criminal subject, we must engage in the study of the limbic system, known as the scene of emotions related to the most primitive part of our neural functioning. The amygdala has the function of activating emotions and has the ability to stimulate both predatory and affective attacks. The hippocampus has a role in modulation and aggressiveness adjustment, and with adequate activation it is able to trigger a predatory attack. The thalamus has the function of connecting and sorting between the emotional limbic areas and the regulatory cortical brain areas. The midbrain, once activated, has the ability to manage an affective aggression with an emotional component. This brain mechanism, now explored with neuroscientific techniques, has allowed comparison of subcortical activation in criminal murderers, with subjects in 3 categories: reactive, proactive, and control group. These findings have confirmed a higher level of activation in the subcortical limbic regions of the first two groups, compared to the control group.

This activation was more evident in the right cerebral hemisphere, which is more evidently connected with emotions. From the evidence presented so far, one can assume that cold-blooded murderers have preserved prefrontal functions, rendering them capable of regulating their actions, with the consequence of being able to perform criminal acts in a relatively cautious manner and with premeditation $[8,9]$. More and more interest is being raised by the concrete possibility to study the areas posterior to the frontal region. The angular gyrus, defined as area 3 in the map created by anatomist Korbinian Brodmann, is the brain region in the lower part of the parietal lobe above the temporal cortex, and frontal to the visual cortex. A significant position for the ability to join three of the four main lobes. Neuroimaging techniques with measurement of glucose metabolism revealed that, in these areas, metabolic activity is reduced in criminals compared to a control group.

The impaired functioning of the angular gyrus acts as a threshold in basic skills such as reading, writing, and counting. These limits trigger a slowdown in the learning stages. This aspect, which is correlated with a compromised educational and professional history, is typically part of a violent criminal personality. Neuroscientific imaging of the hippocampus and peripheral convolutions has allowed the demonstration of abnormalities in areas previously connected to criminal behavior. This evidence has been particularly linked to antisocial behavior. In Sweden, Henrik Soderstrom [10] confirmed a reduced hippocampal function in violent criminals with psychopathy. In the United States Kent Kiehl suggested a relationship between hippocampus and psychopathy [11].

\section{PET scanner}

Positron Emission Tomography is an analytical imaging technique, utilizing positron-emitting radioisotope-marked compounds as molecular probes to obtain images and measure biochemical processes in vivo in mammal biology. Physical Principles [12]: The radionuclides utilized in PET are substances containing an excess of protons: they are energetically unstable, and tend to decay spontaneously to reach a greater nuclear energetic stability by emitting positrons. These are electron anti-matter, i.e., particles with equal mass and opposite electric charge. Positrons are emitted with a variable kinetic energy content, called 'positron range', and they are able to travel few millimeters. Once all kinetic energy is dissipated and the condition of kinetic rest is reached, a positron interacts with an electron; the effect is the disappearance of both particles' masses (annihilation), and by the principle of conservation of energy, the formation of two annihilation photons of $511 \mathrm{KeV}$ (the energy of $511 \mathrm{KeV}$ of each photon is equivalent to the resting mass of an electron or a positron). The two photons, or gamma rays to be precise, depart from the annihilation point with equal velocities (close to the speed of light) and opposite directions, thus forming a line named 'annihilation line'. The annihilation photons reach the tomograph almost simultaneously and strike a pair of detectors, which are positioned opposite one another and joined by a computerized coincidence circuit. When a photon strikes the crystal within the detector, light is emitted by scintillation, amplified by a photomultiplier, and converted into an electrical signal. When the computer registers two electrical charges in a time interval of 5-20 nanoseconds (coincidence interval), it encodes an annihilation 
event. The line that joins the detectors is called 'line of coincidence': it coincides with the line of annihilation and passes through the point of annihilation. Each annihilation event produces a coincidence line. Radionuclides are artificially produced by a linear particle accelerator, called a ciclotron. The main radionuclides available today for PET studies are ${ }^{18} \mathrm{~F}$ (used as a substitute for hydrogen, as this latter cannot emit positrons), ${ }^{14} \mathrm{O},{ }^{15} \mathrm{O},{ }^{11} \mathrm{C},{ }^{13} \mathrm{~N}$; positron-emitting nuclides of $\mathrm{Cu}, \mathrm{Zn}$, $\mathrm{K}, \mathrm{Br}, \mathrm{Rb}, \mathrm{I}, \mathrm{P}, \mathrm{Fe}, \mathrm{Ga}$ are also used. Radionuclides are utilized to mark a substance, and allow for in vivo measurements of a physiological variable connected to the kinetic or dynamic characteristics of this substance. Prior knowledge of the kinetic and dynamic properties of the substance to be marked is required: radionuclides determine modest and correctable variations of kinetic properties ("isotope effect") but do not modify physiological or biochemical properties of the substance to be marked. The introduction of radionuclides inside the human organism can take place either intravenously $\left(\mathrm{H}_{2}{ }^{15} \mathrm{O} ;{ }^{18}\right.$ Fluoro-2-deoxyD-glucose) or by inhalation $\left(\mathrm{C}^{15} \mathrm{O}_{2}\right)$, depending on the kinetic and physical properties of the marked substance. PET images have allowed identification of the neural network of the hippocampus implicated in the appreciation of social objectives. As a result we come to assume a link between socially inadequate or even violent behavior justified by the inability to evaluate ambiguous stimuli, and social situations that can stimulate a violent act. Exciting results have been obtained through the study of regional cerebral blood flow by PET, in relation to criticism, judgment, and control of behavior. This type of brain activity has been extensively evaluated in patients with post-traumatic deficits or degenerative diseases. Subjects who have an intact ability to understand, but are not able to control some impulses, display an abnormality that makes them unable to perceive the emotions of others.

In this context the study on mirror neurons is particularly interesting: these anticipate and understand not only motor tasks, but also the emotions involved in the motor moment. New neuroimaging techniques are now able to objectively reveal the neurobiological components of decision-making behavior, involuntary activity and reactivity, up to the possibility of documenting the neural basis of moral judgment. In some subjects it is possible to verify the inability to block certain automatic responses. In this specific context there is a significant increase in knowledge of the activity of mirror neurons, in relation to neurobiological activity of certain brain areas. Environmental components, genetics, post traumatic events, or recent-onset diseases are not to be deemed insignificant; however, the impaired brain mechanism that causes and sustains an inappropriate behavior or wrongdoing is often identifiable. It is possible, for example, to distinguish between violent and non-violent schizophrenics, mild and severe personality disorder: these diagnostic opportunities are given by the possibility of using Voxel-Based Morphometry (VBM).

\section{Metabolism of amines}

Several studies of molecular biology and behavioral genetics are of great interest with regard to the identification of genetic influence on behavior and personality. Of particular interest is the attention paid to the study of susceptibility of the MAO-A gene [13]. MAO are classified as oxidoreductases and catalyze the following reaction:

$$
\begin{array}{ll}
\mathrm{H} & \mathrm{H} \\
\mid & \mathrm{I}-\mathrm{C}-\mathrm{NH}_{2}+\mathrm{O}_{2}+\mathrm{H}_{2} \mathrm{O} \\
\text { I } & \mathrm{R}-\mathrm{C}=\mathrm{O}+\mathrm{NH}_{3}+\mathrm{H}_{2} \mathrm{O}_{2} \\
\mathrm{H}
\end{array}
$$

The genes that code for MAO have been identified on the short arm of the $\mathrm{X}$ chromosome, and they have been encoded as two distinct categories: MAO-A and MAO-B. Neurons and astrocytes appear to be the major locations of such monoamines in the central nervous system. To date we are certain a relationship exists between such monoamines and the neutralization of monoaminergic neurons. More specifically serotonin appears to be oxidized by MAO-A, while MAO-B is able to interfere in the oxidation of phenylethylamine. Oxidations interfere with the activity of adrenaline, noradrenaline and dopamine. The relationship to psychiatric disorders is therefore evident, and is extensively studied in antisocial disorders, attention deficit disorder, social phobias and substance abuse.

\section{Neurosciences and justice}

What is described here is also particularly interesting from a legal point of view, not only in relation to the purely diagnostic aspect of diseases, but especially with regard to a judgment of imputability, where an exclusively descriptive diagnosis loses its effectiveness. For this legal aspect it is only right to refer to the United Sections, which have subordinated the criminalization of personality disorders to quantitative data indicating amount, intensity, and severity of the disorder. The expert who intends to make a valuable contribution, must be able to "measure" intensity and severity of the disorder. A higher value "of the measure" obtained will determine a greater value of the expert's evidence. The concept of mental illness then needs to change and include in its definition the presence of structural abnormalities of the brain, and/or its receptors, or the presence of other pathological changes. We will need to take note of the inability of the subject to manage empathy, emotions, and impulsivity, even while having intact cognitive abilities. Neurosciences could then extend their implications in criminal cases beyond the concept of imputability. Some of the applications will deal with the evaluation of testimonials and declarations of innocence: thanks to fMRI, SPECT techniques, VBM [14], it is now possible to uncover a lie in $90 \%$ of examined cases. Some areas of application will concern the assessment of testimony or statements of innocence. Through functional magnetic resonance today it is possible to ascertain a lie in $90 \%$ of cases examined. In the United States it is customary to assign a specific hearing in order to establish the adequacy of the acquisition of confession; in the UK the Home Office has set appropriate guidelines for the interrogation of a minor. In such contexts neurosciences find implications in the possibility of assessing the ability to stand trial and the formation of evidence in general, as well as scientific validation of the implications regarding the validity of consent. Neurosciences based on an experimental method, with the possibility of an external check of reliability, acquire greater value compared to other classical psychological approaches. It is essential that specialists in the same neuroscience field always require an assessment of the scientific nature of the discipline that generates the test, the admissibility of evidence and, when produced, its results.

\section{References}

1. Huettel SA, Song AW, McCarthy G (2004) Functional Magnetic Resonance Imaging Sinauer Associat ISBN 0-87893-288-7.

2. Ashburner J, Friston KJ, Giugno (2000) Voxel based morphometry-The metods Neurolmage 11: 805-821 [Crossref].

3. Watkins KE, Paus T, Lerch JP, Zijdenbos A, Collins DL, et al. (2001) Structural Asymetries in the Human Brain: a Voxel-basewd Statistical Analysis of $142 \mathrm{MRI}$ Scans. Cereb Cortex 11: 868-877. [Crossref]

4. Luders E, Gaser C, Jancke L, Schlaug G (2004) A voxel-based approach to gray matter asymmetries. Neuroimage 22: 656-664. [Crossref] 
5. Blaschko H (1963) Amineoxidase. In: Boyer PD, Lardy H, Myrbäck K (Eds), TheEnzymes (2ndedn), 8, Academic Press, New York, 337-351.

6. Damasio A (1995) Descartes' Error: Emotion, Reason, and the Human Brain,GP Puntnam's Sons, New York, 1994, trad. it. Di Macaluso, F. L'errore di Cartesio, Emozione, ragione e cervelloumano, Adelphi, Milano.

7. Bechara A, Damasio AR (2005) The somatic marker hypothesis: A neural theory of economics decision. In: "Games and Economic Behavior" 52: 336-72.

8. Elliot FA (1992) Violence: The neurologic contribution:An overview. Arch Neurol49: 595-603. [Crossref]

9. Adamec RE (1991) Role of the temporal lobe in feline aggression and defense. Special Issue: Ethoexperimental psychology of defense: Behavioral and biological processes. Psychological Record 41: 233-53.

10. Mirsky AF, Siegel A (1994) The neurobiology of violence and aggression. In: "Understanding and Preventing Violence", vol. 2, Biobehavioral Influences, a cura di,
Reiss AJ, Miczek KA, Roth JA(Eds), National Academy Press Washinton, D.C., 159-72.

11. Soderstrom H, Tullinberg M, Wikkelso C, Ekholm S, Forsman A (2000) Reduced regional cerebral blood flow in non-psycotic violent offerders. Psychiatry Res 98: 2941. [Crossref]

12. Rubia K, Halari R, Smith AB, Mohammed M, Scott S, et al. (2008) Dissociated funcional brain abnormalities of inhibition in boys with pure conduct disorder and in boys with pure attention deficit hyperactivity disorder. Am J Psychiatry 165: 889-97. [Crossref]

13. Metodologie per lo studio in vivo del metabolismocerebrale Unversità di Pisa Istituto Bioclinica.

14. Sanford HK, Paulsen MG (1975) Criminal law and its processes: Cases and materials Little, Brown.

15. Psychiatry Neuroimaging Laboratory, Department of Psychiatry, Brigham and Women's Hospital, Harvard Medical School, Boston, MA, USA.

Copyright: $(02016$ Posa F. This is an open-access article distributed under the terms of the Creative Commons Attribution License, which permits unrestricted use, distribution, and reproduction in any medium, provided the original author and source are credited. 